\title{
Determinación de la capacidad conservante del aceite esencial de canela sobre uvilla (Physalis peruviana) como tratamiento postcosecha
}

\section{Determination of the preservative capacity of cinnamon essential oil on uvilla} (Physalis peruviana) as a post-harvest treatment

González Cabrera María Verónica. ${ }^{1}$, Tatiana Elizabeth Sánchez Herrera. ${ }^{2}$, \& Armando Vinicio Paredes Peralta. ${ }^{3}$

\begin{abstract}
. DOI: https://doi.org/10.33262/concienciadigital.v3i2.1.1235

Andean fruits such as uvilla (Phisalys peruviana) have become a consumption trend due to their organoleptic and nutritional properties, however, like other fruits during the postharvest stage, the main deterioration experienced is due to the action of microorganisms mainly mushrooms. For this reason, the present research values cinnamon essential oil as a potential bioconservative. Cinnamon oil was evaluated to determine its antifungal activity "in vitro" against Botritys sp asyle of uvilla post harvest, in a completely randomized design from which results of fungicidal activity were obtained from $250 \mathrm{ppm}$ in Papa Dextrose Agar culture medium PDA and a fungistatic effect using a concentration of $125 \mathrm{ppm}$ of cinnamon essential oil. Similarly, the preservative activity was determined "in situ" through physicochemical, microbiological and sensory tests on fresh fruit stored under two temperature conditions $\left(5^{\circ} \mathrm{C}\right.$ and $\left.21^{\circ} \mathrm{C}\right)$; using the immersion method in solutions with concentrations of 250 and $500 \mathrm{ppm}$ of cinnamon essential oil. In general, the treatments helped to preserve the product better, giving us a value of 12 days of shelf life, delaying the growth of molds and yeasts, allowing the optimal organoleptic characteristics of the uvilla fruit to be maintained.
\end{abstract}

\footnotetext{
${ }^{1}$ Escuela Superior Politécnica de Chimborazo, Facultad de Ciencias Pecuarias. Riobamba, Ecuador. mariav.gonzalez@espoch.edu.ec

${ }^{2}$ Escuela Superior Politécnica de Chimborazo, Facultad de Ciencias Pecuarias. Riobamba, Ecuador. tatiana.sanchez@espoch.edu.ec

${ }^{3}$ Escuela Superior Politécnica de Chimborazo, Facultad de Ciencias Pecuarias. Riobamba, Ecuador. armando.paredes@espoch.edu.ec
} 
Keywords: Essential oil, cinnamon, preservative, uvilla, antifungal, postharvest

\section{Resumen.}

Los frutos andinos como la uvilla (Phisalys peruviana) se han convertido en una tendencia de consumo debido a sus propiedades organolépticas y nutricionales, sin embargo, al igual que otras frutas durante la etapa postcosecha el principal deterioro que experimenta se debe a la acción de microorganismos principalmente hongos. Por esta razón la presente investigación valora al aceite esencial de canela como un potencial bioconservador. El aceite de canela fue evaluado para determinar su actividad antifúngica "in vitro" contra Botritys sp asilado de uvilla post cosecha, en un diseño completamente al azar del que se obtuvieron resultados de actividad fungicida a partir de $250 \mathrm{ppm}$ en medio de cultivo Papa Dextrosa Agar PDA y un efecto fungistático usando una concentración de 125 ppm de aceite esencial de canela. De igual manera se determinó la actividad conservante "in situ" a través de ensayos fisicoquímicos, microbiológicos y sensoriales sobre el fruto fresco almacenado en dos condiciones de temperatura $\left(5^{\circ} \mathrm{C}\right.$ y $\left.21^{\circ} \mathrm{C}\right)$; utilizando el método de inmersión en soluciones con concentraciones de 250 y 500 ppm de aceite esencial de canela. En general, los tratamientos ayudaron a que se conserve mejor el producto, dándonos un valor de 12 días de vida útil retrasando el crecimiento de mohos y levaduras permitiendo mantener las características organolépticas óptimas de los frutos de uvilla.

Palabras claves: Aceite esencial, canela, conservante, uvilla, antifúngico, postcosecha.

\section{Introducción.}

El nombre uvilla o uchuva (Physalis peruviana) corresponde a un fruto perteneciente a la familia de las Solanaceae, cuyo origen se establece en los andes peruanos, presente también en Colombia, Perú, Ecuador y Bolivia (Álvarez, Campoverde y Espinosa, 2012). Presenta un sabor dulce y es muy apreciada debido a su alto contenido de vitaminas A y C; además de contener en su composición microelementos como el hierro y el fósforo que le atribuyen propiedades nutricionales y medicinales. (Almanza, et al., 1993). La uchuva o Uvilla es una especie tolerante, capaz de adaptarse a climas mediterráneos y cualquier tipo de suelo incluso cortezas de árboles o condiciones de poca luz (Brito, 2002). Por tales motivos la uvilla posee una amplia distribución actualmente, siendo Colombia el país número uno en exportar uvilla a nivel mundial seguido por Sudáfrica, Zimbabwe, Kenya, Ecuador, Perú, Bolivia y México (Calvo, 2009, p.2) 
La uvilla es un producto no Tradicional que se expande dentro del sector agrícola del Ecuador y que de acuerdo con datos de PROECUADOR (2016) produce más de 700 hectáreas para exportación. El envío de este tipo de fruta ecuatoriana no tradicional se encuentra enmarcada en la estrategia nacional de incremento a las exportaciones de este tipo de productos agrícolas (AGROCALIDAD, 2019). En la actualidad, la uvilla ha ingresado a 43 mercados alrededor del mundo. Desde el año 2018, este sector logró exportar un total de ciento cincuenta mil dólares siendo sus principales destinos: Francia, Canadá y Países Bajos. De acuerdo con cifras del Banco Central del Ecuador, en el período comprendido entre enero y septiembre 2019, ese rubro aumentó a USD 160 mil dólares. (MIPRO, 2019).

Al constituirse como una fruta exótica muy importante en el mercado internacional, la uvilla, requiere de un manejo técnico que permita a los productores aprovechar estas oportunidades que se presentan en el mercado, es decir cumplir con las normas de calidad y seguridad alimentaria establecidas por los diferentes destinos internacionales a los que el productor quiere alcanzar. (García. et al, 2008). A pesar de los esfuerzos los agricultores enfrentan en etapa postcosecha enfermedades ocasionadas por hongos, bacterias y nematodos. Además, en el mercado se prefiere el consumo de la uvilla sin el capacho (o capuchón) el mismo que cumple una importante función de protección frente a daños mecánicos, físicos o biológicos.

El tiempo de vida útil baja de una manera considerable, cuando se retira el capuchón de la fruta, por lo tanto, es necesario buscar medios o condiciones que protejan a la fruta fresca de manera que se logren tiempos de vida útil similares o mayores a los alcanzados para la fruta con capuchón. La composición y el bajo pH característico de la uvilla, la hace muy sensible diferentes alteraciones originadas por hongos, sobre todo si la humedad, temperatura y tiempo de almacenamiento postcosecha no son los adecuados. (Rodríguez, 2006). Una investigación realizada por el Servicio Ecuatoriano de Sanidad Agropecuaria SESA con el apoyo de la Corporación de Promociones de exportaciones CORPEI, demuestran la presencia de 8 enfermedades causadas por hongos y bacterias entre las que se mencionan: Moho gris (Botrytis sp), mancha gris (Cercospora sp), Mal del semillero (Phytium sp), Fusariosis (Fusarium sp) entre otras (Agrocalidad, 2005).

Las enfermedades y plagas mencionadas en el párrafo anterior han sido controladas por los productores a través del uso de tratamientos con fungicidas químicos, los mismos que constituyen 
un peligro por los residuos que dejan en el producto y que pueden afectar la salud del consumidor. Una alternativa propuesta en varios estudios ha sido la aplicación de tratamientos térmicos que disminuyen la carga microbiana, pero causan cambios en la calidad visual y sensorial del producto con pérdidas de color, firmeza y la pérdida de ciertos compuestos bioactivos como fenoles, flavonoides, fitoalexinas y otros (Shama, 2007). Inclusive en los cultivos de uvilla se ha llegado a aplicar tratamientos con plaguicidas, aun sin la presencia de plagas llevando a evidenciar efectos perjudiciales como resistencia al agroquímico, incremento en costos de producción y afectaciones a la salud del agricultor.

Una alternativa al uso de plaguicidas es la aplicación de sustancias químicas para la conservación de alimentos. Aunque se ha descrito que una gran variedad de agentes químicos posee capacidad conservante solamente una pequeña cantidad han sido permitidos para su uso en los alimentos. Esta situación responde a las rigurosas normas de seguridad señaladas por la FDA y, en menor proporción, al hecho de que cuando se añaden a determinados alimentos, pocas sustancias químicas siguen ejerciendo su actividad conservante (Villada, 2010)

Con estos antecedentes es importante el estudio de innovadores tratamientos pos cosecha que permitan incrementar el periodo de vida útil de los alimentos sin poner en riesgos la salud del consumidor y la calidad de los productos. Las recientes políticas de seguridad alimentaria y tendencia actual de los consumidores por alimentos de origen natural y orgánico abren la posibilidad de que los productos naturales tradicionalmente usados sean una alternativa complementaria (Prakash et al., 2012).

Los productos naturales, han sido utilizados desde la antigüedad como aromatizantes y preservantes de alimentos. Los aceites esenciales son considerados en la actualidad como una alternativa natural para la industria alimenticia ya que poseen actividades antisépticas, antifúngicas, antimicrobianas y aromatizantes. También han sido ampliamente estudiados por su biodisponibilidad, los pocos efectos secundarios o a la toxicidad que puedan causar, así como la mejor biodegrabilidad comparado con antibióticos y preservantes disponibles (Martínez. 2007).

La actividad antimicrobiana que presentan los aceites esenciales se debe a fracciones de su composición química como son los compuestos fenólicos (CCI,2004). De igual manera existen 
estudios que prueban que la capacidad conservante de los aceites esenciales se deriva de terpenoides, flavonoides y fenoles presentes en su composición química. Los aceites esenciales son productos secundarios de las plantas que los usan como un mecanismo de defensa frente a ataques microbianos y de e insectos. De hecho, muchos de estos compuestos han sido usados en forma pura o extractos vegetales como alimento o aplicaciones médicas en humanos (Ávalos et al, 2009).

La composición química del aceite esencial de canela (Cinnamomum zeylanicum) presenta del 75 $-85 \%$ de eugenol como constituyente principal, el mismo que de acuerdo con estudios realizados posee actividad antibacterial, de igual manera el aceite esencial de canela contiene 5\% de cinamaldehído, compuesto químico con carácter aromático y características antimicrobianas. (FAO, 2006), el cinamaldehído ha sido utilizado tradicionalmente como saborizante $\mathrm{y}$ antimicrobiano en los alimentos. Otros componentes de la canela constituyen resinas cianogénicas y ácido hidrociánico, los mismos que han demostrado tener propiedades antibacteriales y taninos generadores de una acción hemostática y astringente (Martínez et al, 2003, citado en Hernandez,2019). Se ha probado también la efectividad del aceite de canela contra bacterias como Listeria monocytogenes, Escherichia coli, Staphylococcus aureus, Enterobacter spp., Lactobacillus spp. y Pseudomonas. Estudios demuestran igualmente que el aceite esencial de canela presenta actividad fungistática en ciertas concentraciones frente al desarrollo de Aspegillus flavus (Montes et al., 1998)

Estos estudios han demostrado el efecto antibacteriano y fungicida del aceite esencial de canela en bacterias patógenas y ciertos hongos, sin embargo se hace necesario la aplicación de estas facultades de manera práctica, por esta razón el objetivo de la presente investigación es comprobar el efecto antifúngico del aceite esencial de canela sobre los hongos causantes del deterioro postcosecha en uvilla almacenada a diferentes temperaturas y tener una alternativa al uso de sustancias químicas utilizadas como tratamientos conservantes en estos frutos

\section{Metodologia.}

\section{Material vegetal}

El material de estudio fue muestreado usando un modelo no probabilístico, esto es un proceso de selección de acuerdo con el grado de madurez de las frutas siguiendo la norma técnica según lo 
establecido en la norma NTE INEN 2485. Frutas frescas. Uvilla. Requisitos. La misma que establece que la madurez de las uvillas puede evaluarse visualmente según su coloración externa (figura 1), que varía de verde a naranja a medida que madura el fruto. (NTE INEN 2485, 2009).

Figura 1. Escala de color de la uvilla - índice de madurez

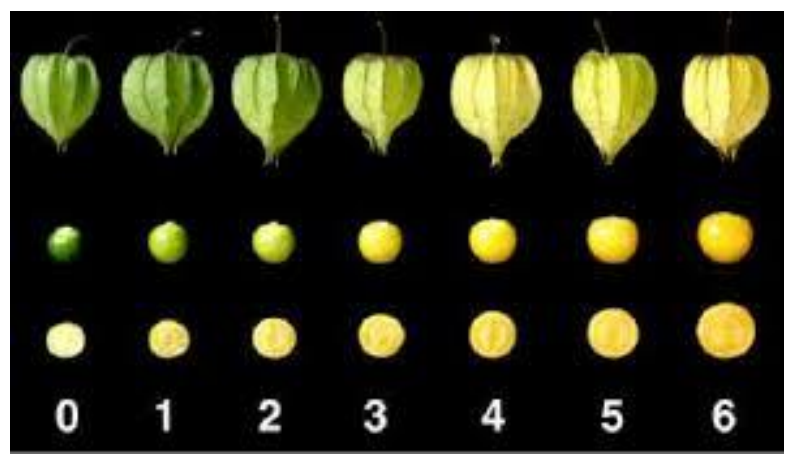

Fuente: NTE 2484:2009. Frutas Frescas: Uvilla. Requisitos

Cumpliendo con los requisitos establecidos en la norma se seleccionaron frutos enteros sin capuchón, sanos sin presentar deterioro, limpios, exentas de plagas que afecten al aspecto general del producto; de consistencia firme, piel suave y brillante. El material vegetal Uvilla (Physalis peruviana) corresponde al ecotipo "ecuatoriana", proveniente de un cultivo comercial del Cantón Chambo, Provincia de Chimborazo. El aceite esencial de Canela (Cinnamomum zeylanicum) con pureza no especificada, grado alimenticio, fue adquirido en la Compañía ISABRUBOTANIK de la ciudad de Ambato, Ecuador.

\section{Caracterización sensorial, física y química del material vegetal seleccionado:}

El material vegetal que fue sometido a una evaluación sensorial a través de la aplicación de escalas hedónicas a través de un panel sensorial de jueces no entrenados (Stone et al, 1993) tomando en cuenta los siguientes aspectos: color, olor, sabor y textura. Para la caracterización física se valoraron aspectos como longitud, diámetro, volumen y peso. La medida de longitud y diámetro se realizó con un vernier marca HOPEZ. Con una balanza de precisión electrónica HR-200, capacidad de $210 \mathrm{~g} \pm 0,0001 \mathrm{~g}$ fue determinado el peso por cada unidad muestreada del material vegetal. Estos procedimientos fueron aplicados de acuerdo con los procedimientos detallados en la NTE 2845. Para la determinación de del contenido de sólidos solubles, $\mathrm{pH}$ y acidez titulable se 
emplearon protocolos señalados en el documento oficial de la A.O.A.C. Official Methods of Analysis, 942.15 (B). La medición de $\mathrm{pH}$ fue realizada mediante un potenciómetro calibrado a una temperatura de $20^{\circ} \mathrm{C}$ y el porcentaje de acidez titulable se reportó como ácido málico. (A.O.A.C.,2000)

\section{Análisis Microbiológico de la fruta fresca (mohos y levaduras):}

A partir de un gramo o centímetro cúbico de la muestra, se procedió a la determinación del número de colonias típicas de levaduras y mohos, para ello se aplicaron los procedimientos establecidos en la norma NTE INEN 1529-11. Determinación de la cantidad de microorganismos mohos y levaduras. Recuento en placa por siembra en profundidad. (NTE INEN 1529-11,2013)

\section{Conteo de colonias}

Se determinaron el número de unidades formadoras de colonias UFC de levaduras por gramo de muestra. Para validar el resultado se procedió a un proceso de aislamiento a través de la técnica de siembra por rayado utilizando una caja de agar PDA y agar extracto de malta. Estas muestras fueron incubadas a una temperatura de $35^{\circ} \mathrm{C}$ por un período de tiempo aproximado de 48 horas. Finalmente se procedió a replicar el conteo de las UFC de hongos/g de las muestras.

\section{Determinación del efecto conservante del aceite esencial de canela (Cinnamomum zeylanicum) sobre uvilla fresca}

Para probar el efecto conservante del aceite esencial de canela se utilizaron frutas cuyo grado de madurez se determinó con con base en la norma NTE INEN 2485. Frutas frescas. Uvilla. Estas fueron sometidas a un proceso de lavado con agua corriente y agua destilada en tres ocasiones. Posteriormente fueron secadas a temperatura ambiente $\left(25 \pm 3^{\circ} \mathrm{C}\right)$. Para cada tratamiento se emplearon 10 frutos como unidad experimental y tres repeticiones de cada una (tabla 1). Se seleccionó al método de inmersión como la técnica para aplicar el aceite esencial de canela sobre la fruta muestral, de esta forma se garantizó evitar pérdidas de la solución. Adicionalmente se consideraron variables como el tiempo de inmersión, concentración del aceite esencial de canela y temperatura de almacenamiento de la fruta recubierta. Se trabajó por duplicado y con dos testigos. 
Tabla 1. Tratamientos utilizados para la determinación de la actividad antimicrobiana del aceite esencial de canela sobre uvilla fresca (Physalis peruviana L.)

\begin{tabular}{cccc}
\hline Tratamiento & $\begin{array}{c}\text { Concentración aceite } \\
\text { esencial de canela }(\mathbf{p p m})\end{array}$ & $\begin{array}{c}\text { Tiempo } \\
(\mathbf{d i ́ a s})\end{array}$ & $\begin{array}{c}\text { Temperatura } \\
\left({ }^{\mathbf{C}} \mathbf{C}\right)\end{array}$ \\
\hline Testigo & & 1 & 21 \\
T1 & 250 & 7 & 21 \\
T2 & 250 & 7 & 5 \\
T3 & 500 & 7 & 21 \\
T4 & 500 & 7 & 5 \\
T5 & 250 & 15 & 21 \\
T6 & 250 & 15 & 5 \\
T7 & 500 & 15 & 21 \\
T8 & 500 & 15 & 5 \\
T9 & 250 & 21 & 21 \\
T10 & 250 & 21 & 5 \\
T11 & 500 & 21 & 21 \\
T12 & 500 & 21 & 5 \\
T13 & 250 & 30 & 21 \\
T14 & 250 & 30 & 5 \\
T15 & 500 & 30 & 21 \\
T16 & 500 & 30 & 5 \\
\hline
\end{tabular}

Elaborado por: Grupo de investigación

Las soluciones utilizadas en el proceso de inmersión se prepararon en concentraciones de 250 y 500 ppm. Se utilizaron $50 \mathrm{mg}$ del Aceite esencial de Canela y disuelto en $500 \mu \mathrm{L}$ de Tween 20 como disolvente teniéndose una concentración final de 100000 ppm. A partir de esta solución madre se realizaron diluciones al décimo con concentración finales de 500 y 250 ppm y se llevaron a un volumen final de $500 \mathrm{~mL}$.

El proceso de inmersión se realizó utilizando vasos de precipitación con las soluciones preparadas de 250 ppm y 500 ppm de aceite esencial de canela, sumergiendo las muestras de uvilla durante 5 segundos en las soluciones respectivas. El procedimiento de secado se llevó a cabo bajo una campana de flujo laminar durante 5 minutos, tanto para las muestras recubiertas como para las uvillas control que fueron sumergidas en agua destilada estéril.

Una vez secadas, las muestras uvilla con recubrimiento de aceite esencial de canela, se ubicaron 10 unidades muestrales en recipientes de plástico, uno para cada tratamiento, respetando una distancia de 2 a $3 \mathrm{~cm}$ entre cada muestra. Los recipientes plásticos fueron almacenados de manera 
aleatoria bajo equivalentes condiciones de temperatura y humedad. Las condiciones de almacenamiento fueron dos: $21^{\circ} \mathrm{C}$ (temperatura ambiente) y $5^{\circ} \mathrm{C}$ (temperatura de refrigeración). Las muestras de cada tratamiento se almacenaron por triplicado y las temperaturas se monitoreaban de manera constante con el uso de un termómetro de mercurio. Al mismo tiempo cada recipiente era cambiado de posición para garantizar que durante el experimento las condiciones de temperatura y humedad eran equivalentes para todas las muestras.

El efecto consérvate del aceite esencial de canela sobre uvilla en la etapa de postcosecha se determinó a través de la cuantificación de varios parámetros entre los que constan: pH, porcentaje de acidez titulable; indicadores de análisis sensorial: textura, color, olor y sabor, además del recuento de mohos y levaduras. Los resultados obtenidos en el presente estudio fueron analizados con un diseño experimental completamente al azar en arreglo simple. Los resultados obtenidos se procesaron y analizaron en el programa SPSS STATISTICS, bajo el modelo ANOVA y el uso de la Prueba T para muestras independientes además de la Prueba de Chi cuadrado.

\section{Resultados.}

Los datos que se reportan fueron obtenidos sobre la base de la metodología indicada.

\section{Medida de variables fisicoquímicas y microbiológicas en uvilla fresca (Physalis peruviana l.) bajo dos condiciones de almacenamiento postcosecha}

La fruta cosechada fue sometida a dos condiciones de almacenamiento: temperatura de $21^{\circ} \mathrm{C}$ con una humedad relativa del $67 \%$ y temperatura de $5^{\circ} \mathrm{C}$ con una humedad relativa de $90 \%$, estos datos servirán como testigo para establecer una comparación con los resultados obtenidos en la fruta tratada con aceite esencial de canela. En la Tabla 2 se presentan los resultados obtenidos en la medición de indicadores sensoriales: olor, color, sabor y textura, parámetros químicos: $\mathrm{pH}$, acidez titulable y análisis microbiológicos: recuento de mohos y levaduras.

Tabla2: Resultados de acidez, $\mathrm{pH}$, temperatura y unidades propagadoras (up/g) para uvilla (Physalis peruviana I.)

\begin{tabular}{|c|c|c|c|c|}
\hline $\begin{array}{l}\text { Tratamie } \\
\text { nto }\end{array}$ & $\begin{array}{l}\text { Tiempo } \mathrm{T}\left({ }^{\circ} \mathrm{C}\right) \\
\text { (días) }\end{array}$ & Características sensoriales & Pruebas Físicas & $\begin{array}{c}\text { Análisis } \\
\text { microbiológico }\end{array}$ \\
\hline
\end{tabular}


Vol. 3, $\mathrm{N}^{\circ} 2.1$, p. 210-230, mayo, 2020

\begin{tabular}{cccccccccc}
\hline & & & Color & Text & Olor & Sabor & pH & $\begin{array}{c}\text { \% } \\
\text { Acidez }\end{array}$ & $\begin{array}{c}\text { Recuento } \\
\text { mohos y lev. } \\
\text { (UP/g) }\end{array}$ \\
\hline Testigo & 1 & & & & & & & & 50 \\
T1 & 7 & 21 & 10 & 10 & 10 & 10 & 4.06 & 1.95 & 52 \\
T2 & 15 & 21 & 10 & 10 & 10 & 10 & 4.44 & 1.77 & 58 \\
T3 & 21 & 21 & 0 & 0 & 0 & 0 & 4.90 & 1.64 & 65 \\
T4 & 30 & 21 & 0 & 0 & 0 & 0 & 3.95 & 1.54 & 60 \\
T2 & 7 & 5 & 10 & 10 & 10 & 10 & 4.10 & 1.94 & 50 \\
T4 & 15 & 5 & 10 & 10 & 10 & 10 & 4.32 & 1.68 & 51 \\
T6 & 21 & 5 & 10 & 10 & 10 & 10 & 4.73 & 1.56 & 55 \\
T8 & 30 & 5 & 0 & 0 & 0 & 0 & 3.75 & 1.48 & 48 \\
\hline
\end{tabular}

Elaborado por: Grupo de investigación

La apariencia general de la fruta muestral muestra cambios de alrededor del $40 \%$ en temperatura de $5^{\circ} \mathrm{C}$ (refrigeración) y aproximadamente del $60 \%$ cuando se almacenaron a temperatura ambiente $\left(21^{\circ} \mathrm{C}\right)$. Las muestras almacenadas a $21^{\circ} \mathrm{C}$ presentaron síntomas de alteración como la presencia de mohos y levaduras, al día 18, y se incrementó en días posteriores, llegando a su valor máximo en el día 21 como se observa en la Tabla 2. Con respecto a los análisis realizados para las muestras almacenadas a temperatura de refrigeración $5^{\circ} \mathrm{C}$, se observan señales de alteración y deterioro por la acción de mohos y levaduras en el día 28, con un incremento mínimo en días posteriores. De igual manera se pudo determinar que a temperatura de refrigeración no se observaron cambios en la apariencia de la fruta hasta el día 25. Para las muestras de uvilla postcosecha parámetros sensoriales olores no característicos, sabores extraños y presencia de manchas oscuras en la fruta, registraron los mayores porcentajes de cambio a partir de la cuarta semana de estudio y en la temperatura más alta de almacenamiento es decir a $21^{\circ} \mathrm{C}$.

En lo que respecta a la medida del $\mathrm{pH}$, durante la primera y segunda semana del ensayo los frutos mantuvieron un $\mathrm{pH}$ de 4,22 a 4,44, respectivamente. Al finalizar el ensayo (cuarta semana) el pH disminuyó considerablemente con relación a las mediciones anteriores, lo cual concuerda con investigaciones realizadas previamente que registraron un incremento en el valor del $\mathrm{pH}$ a partir del día 16 de almacenamiento y su posterior reducción. (Novoa et al, 2006)

El incremento progresivo del $\mathrm{pH}$ hasta la tercera semana de estudio puede considerarse una consecuencia de la disminución de ácidos presentes en la pulpa del fruto, la disminución a partir de la cuarta semana de estudio podría ser atribuida a procesos de acidificación durante la 
senescencia del fruto. En lo que respecta a los valores de acidez total titulable (\%) con el proceso de maduración se observa un descenso uniforme lo que coincide con relaciones similares reportadas en estudios realizados en la pitahaya (Gallo, 1993). La disminución del valor de la acidez puede interpretarse ya que los ácidos en el fruto son utilizados como substrato de respiración, ya que al igual que los carbohidratos, son fuentes de carbono e hidrógeno. (Rodríguez, 2003). En la uvilla, la mayor proporción de los ácidos la constituye el ácido cítrico, cuando se ha alcanzado el máximo de acumulación del almidón se presenta una disminución del contenido de los ácidos por tal motivo se considera que la acidez titulable decrece en cuanto los ácidos orgánicos se convierten en sustratos de la respiración. (Fischer y Lüdders, 1997), lo cual concuerda con lo observado durante el ensayo.

Una de las respuestas experimentales de la presente investigación constituyó el el recuento microbiano de mohos y levaduras, el que se realizó mediante siembras periódicas a partir de los días: cero, siete, quince, veinte y treinta. De acuerdo con lo señalado en el reglamento sanitario de los alimentos el límite para el recuento de mohos y levaduras es de 105 UFC/g (López 1999), el valor inicial reportado para las muestras de uvilla en el recuento de mohos corresponde a 50ufc/g. A pesar de encontrarse dentro de un rango establecido las condiciones de almacenamiento como la temperatura llevó a que exista un incremento de carga microbiana que puede acelerar el deterioro de la calidad e inocuidad de la uvilla postcosecha.

Aunque no se puede establecer una relación estadística entre el desarrollo de mohos y levaduras con respecto al incremento de acidez y diminución del valor del $\mathrm{pH}$, puede determinarse que la actividad metabólica de los microorganismos causales del deterioro de la fruta permite la transformación de azúcares a ácidos orgánicos de la fruta lo que influye directamente en la disminución del $\mathrm{pH}$ y el incremento de la acidez titulable. En las muestras almacenadas a $5^{\circ} \mathrm{C}$ (temperatura de refrigeración), no se pudo observar un desarrollo considerable de mohos y levaduras; sin embargo, se podía determinar visualmente que en la superficie de la fruta existían manchas y áreas húmedas a causa del consumo de sustrato e inicio del crecimiento de los hongos

Determinación del efecto conservante del aceite esencial de canela sobre uvilla fresca (Physalis peruviana l.) por el método de inmersión. 
La aplicación de un recubrimiento de aceite esencial de canela sobre las unidades muestrales de uvilla fresca, permitieron una mejor conservación del producto dando un resultado de 12 días de vida de anaquel o shelf life sin presentar variaciones en sus características físicoquímicas, sensoriales y microbiológicas, comparados con las muestras testigo sin recubrimiento de aceite esencial. De acuerdo con investigaciones documentales, las uvillas si se almacena con su cáliz, se pueden conservar durante 15 días aproximadamente manteniéndola a una temperatura de 17 a $19^{\circ} \mathrm{C}$ con una humedad relativa cercana al 70\%. Este tiempo puede prolongarse a más de un mes si la temperatura que se usa está entre 4 y 6 grados centígrados. (Betancourt, 2010).

A partir del día 15 , los tratamientos almacenados a temperatura ambiente $\left(21^{\circ} \mathrm{C}\right)$, presentaron alteraciones sensoriales en su textura y la presencia de una cantidad mínima de microorganismos visibles en 2,5\% de fruta (tabla 3). En similares condiciones se evidenció que un 2,5\% de la fruta recubierta presentaba sabores atípicos, que se atribuyen a la presencia del aceite esencial de canela, ya que estos cambios sensoriales eran notorios mientras mayor era la concentración de aceite esencial. Otro de los inconvenientes presentados fue que las semillas y el centro de la drupa cambiaron de color puesto que se observó un oscurecimiento. Con los resultados reportados se establece que del día 1 al 12 las muestras de uvilla tratadas con aceite esencial de canela no evidenciaron ataque de microorganismos, ni ablandamiento, es decir mantenían sus atributos de calidad e inocuidad, convirtiéndolas en aceptables. Bajo las condiciones de almacenamiento en temperatura de refrigeración $\left(5^{\circ} \mathrm{C}\right)$, las muestras de fruta (uvilla) analizadas no presentaron cambios sensoriales, químicos o microbiológicos manteniendo su calidad y aceptabilidad hasta el día 30 del experimento.

Tabla3: Resultados de acidez, $\mathrm{pH}$, temperatura y unidades propagadoras (up/g) para uvilla (Physalis peruviana I.) con tratamiento de aceite esencial de canela

\begin{tabular}{|c|c|c|c|c|c|c|c|c|c|c|}
\hline \multirow{2}{*}{ Trat } & \multirow{2}{*}{$\begin{array}{l}\text { Concentración } \\
\text { de aceite } \\
\text { (ppm) }\end{array}$} & \multirow[t]{2}{*}{$\begin{array}{l}\text { Tiempo } \\
\text { (días) }\end{array}$} & \multirow[t]{2}{*}{$\begin{array}{c}\mathrm{T} \\
\left({ }^{\circ} \mathrm{C}\right)\end{array}$} & \multicolumn{4}{|c|}{ Características sensoriales } & \multicolumn{2}{|c|}{$\begin{array}{l}\text { Pruebas } \\
\text { Físicas }\end{array}$} & \multirow{2}{*}{$\begin{array}{c}\text { Análisis } \\
\text { microbiológico } \\
\text { Recuento } \\
\text { mohos y lev. } \\
\text { (UP/g) }\end{array}$} \\
\hline & & & & Color & Text & Olor & Sabor & pH & $\begin{array}{c}\% \\
\text { Acidez }\end{array}$ & \\
\hline Testigo & & 1 & & 10 & 10 & 10 & 10 & 4,06 & 1,95 & 50 \\
\hline $\mathrm{T} 1$ & 250 & 7 & 21 & 10 & 10 & 10 & 10 & 4,55 & 1,81 & 24 \\
\hline $\mathrm{T} 2$ & 250 & 7 & 5 & 10 & 10 & 10 & 10 & 4,5 & 1,78 & 20 \\
\hline $\mathrm{T} 3$ & 500 & 7 & 21 & 10 & 10 & 10 & 10 & 4,53 & 1,8 & 32 \\
\hline
\end{tabular}


Vol. 3, $\mathrm{N}^{\circ} 2.1$, p. 210-230, mayo, 2020

\begin{tabular}{lllllllllll}
\hline $\mathrm{T} 4$ & 500 & 7 & 5 & 10 & 10 & 10 & 10 & 4,51 & 1,79 & 28 \\
$\mathrm{~T} 5$ & 250 & 15 & 21 & 10 & 10 & 10 & 10 & 4,58 & 1,75 & 22 \\
$\mathrm{~T} 6$ & 250 & 15 & 5 & 10 & 10 & 10 & 10 & 4,55 & 1,77 & 20 \\
$\mathrm{~T} 7$ & 500 & 15 & 21 & 10 & 10 & 10 & 10 & 4,56 & 1,78 & 32 \\
$\mathrm{~T} 8$ & 500 & 15 & 5 & 10 & 10 & 10 & 10 & 4,54 & 1,79 & 30 \\
$\mathrm{~T} 9$ & 250 & 21 & 21 & 10 & 10 & 10 & 10 & 4,62 & 1,73 & 22 \\
$\mathrm{~T} 10$ & 250 & 21 & 5 & 10 & 10 & 10 & 10 & 4,58 & 1,77 & 19 \\
$\mathrm{~T} 11$ & 500 & 21 & 21 & 10 & 10 & 10 & 10 & 4,6 & 1,75 & 32 \\
$\mathrm{~T} 12$ & 500 & 21 & 5 & 10 & 10 & 10 & 10 & 4,56 & 1,78 & 30 \\
$\mathrm{~T} 13$ & 250 & 30 & 21 & 10 & 10 & 10 & 10 & 4,63 & 1,72 & 24 \\
$\mathrm{~T} 14$ & 250 & 30 & 5 & 10 & 10 & 10 & 10 & 4,58 & 1,74 & 21 \\
$\mathrm{~T} 15$ & 500 & 30 & 21 & 0 & 0 & 0 & 0 & 4,62 & 1,71 & 34 \\
$\mathrm{~T} 16$ & 500 & 30 & 5 & 10 & 10 & 10 & 10 & 4,6 & 1,76 & 32 \\
\hline
\end{tabular}

Elaborado por: Grupo de investigación

\section{pH}

El pH promedio de las muestras control fue de 4,06 al inicio del período de almacenamiento. La aplicación del aceite esencial de canela en concentraciones de 250 y 500 ppm, modificaron el pH de la fruta fresca de manera inicial, incrementándolo debido a las características químicas propias del aceite. Al finalizar el período de almacenamiento, se pudieron determinar valores mayores de $\mathrm{pH}$ con respecto a la fruta control. Los tratamientos almacenados a $5^{\circ} \mathrm{C}$ evidenciaron un incremento menor del $\mathrm{pH}(0.04$ unidades $)$ con respecto a los tratamientos almacenados a $21^{\circ} \mathrm{C}$; observándose un incremento superior en la fruta recubierta con $250 \mathrm{ppm}$ a una temperatura ambiente $\left(21^{\circ} \mathrm{C}\right)$ igual a 0.07 unidades.

Gráfico1: pH vs tiempo de almacenamiento para la uvilla recubierta con aceite esencial

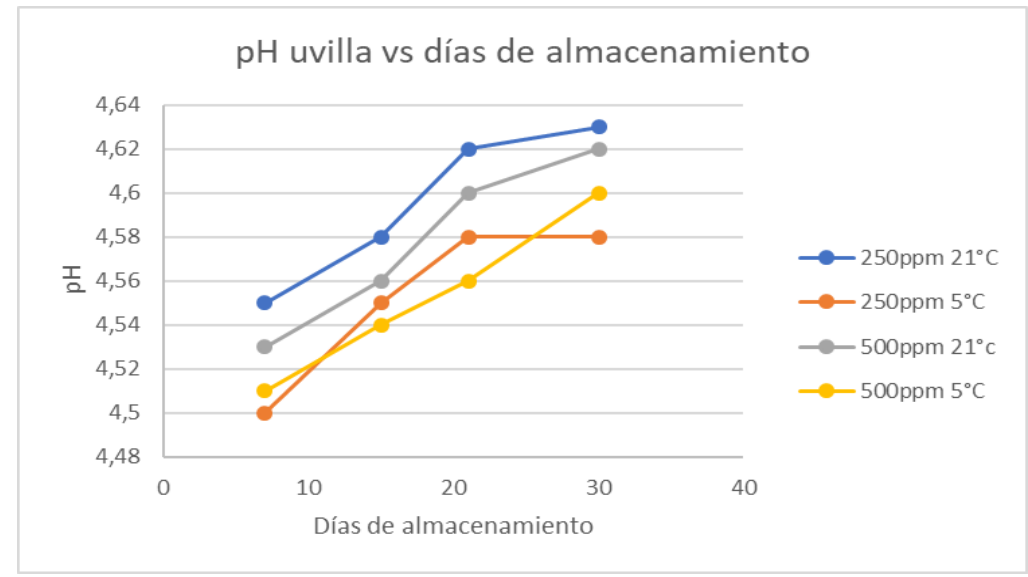

Elaborado por: grupo de investigación 
Las frutas muestrales del experimento tratadas por el método de inmersión en aceite esencial de canela y almacenadas a $5^{\circ} \mathrm{C}$ y $21^{\circ} \mathrm{C}$, experimentaron incrementos menores en el valor del $\mathrm{pH}$ con respecto a los resultados obtenidos para fruta control, como se puede observar en el gráfico 2.

Gráfico 2: Valor de pH en uvillas con y sin recubrimiento de aceite esencial de canela

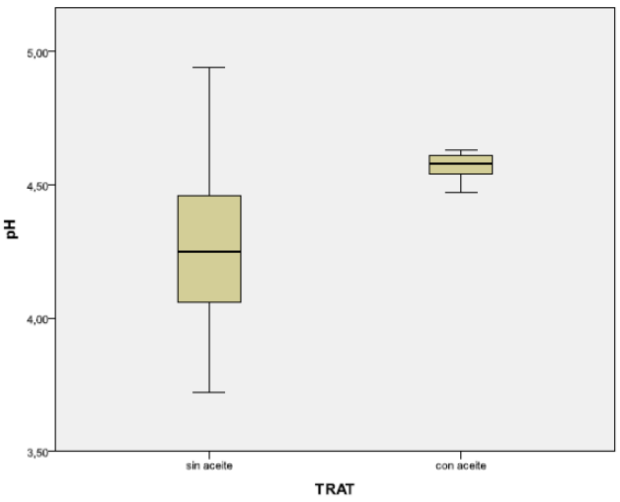

Elaborado por: Grupo de investigación

De acuerdo con la Prueba de T para muestras independientes, se observan diferencias significativas $(\mathrm{p}=0,01)$ entre las muestras de uvillas tratadas con aceite esencial de canela y uvillas frescas sin tratamiento de aceite (tabla4). Estos resultados nos llevan a determinar que la aplicación del aceite esencial de canela tiene un efecto conservante sobre las muestras de uvilla del experimento retardando la senescencia del producto e incrementando la vida útil del mismo.

Tabla4: Prueba t de muestras independientes para $\mathrm{pH}$ uvilla

$$
\begin{gathered}
\text { Prueba de Levene } \\
\text { para la igualdad de }
\end{gathered}
$$

varianzas Prueba T para la igualdad de medias

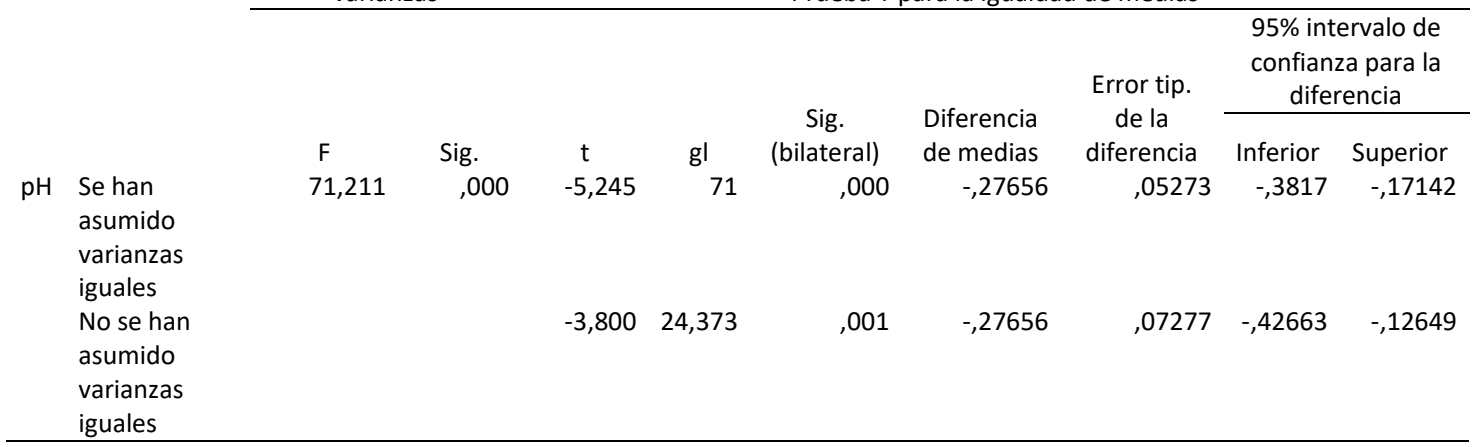

Elaborado por: Grupo de investigación

\section{Porcentaje de Acidez Titulable}

Al comparar los valores de porcentaje de acidez titulable de la uvilla fresca con tratamiento de aceite esencial en dos concentraciones 250 y $500 \mathrm{ppm}$, que fueron almacenadas en dos temperaturas $5^{\circ} \mathrm{C}$ y $21^{\circ} \mathrm{C}$, se puede observar que existe una disminución progresiva del valor de la acidez, sin embargo, esta disminución es menor que la observada para la fruta fresca sin recubrimiento de 
aceite esencial de canela. Los valores de Acidez obtenidos para las uvillas con recubrimiento se observan en el gráfico3, en donde también se puede determinar que en el transcurso del almacenamiento en temperaturas de refrigeración para los tratamientos con aceite esencial de canela existe una disminución menor del valor de la acidez con respecto a los tratamientos que fueron almacenados a temperatura ambiente.

Gráfico3: Evaluación del porcentaje de acidez titulable vs tiempo de almacenamiento para muestras de uvilla tratas con aceite esencial de canela

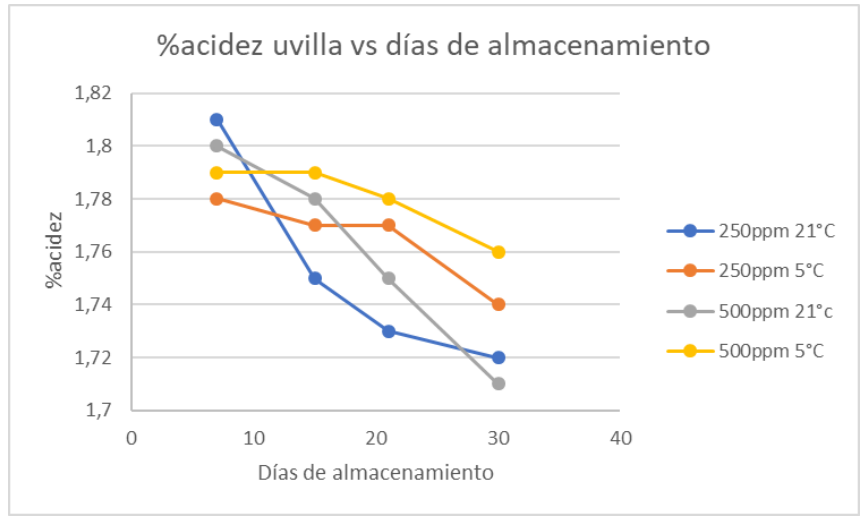

Elaborado por: grupo de investigación

El porcentaje de acidez titulable en la uvilla presenta un decremento en función del tiempo de almacenamiento, como se observa en el Gráfico3. Con respecto a la fruta control el Gráfico 4 evidencia que no existen diferencias significativas al comparar el tratamiento de $250 \mathrm{ppm}$ de aceite esencial de canela con el tratamiento de 500 ppm, al contrario de lo que se puede determinar entre los tratamientos de uvilla sin recubrimiento y con recubrimiento de aceite esencial de canela como se observa en el gráfico 4.

Gráfico4: Comparación del \% de acidez titulable entre muestras control y muestras recubiertas con aceite esencial de canela

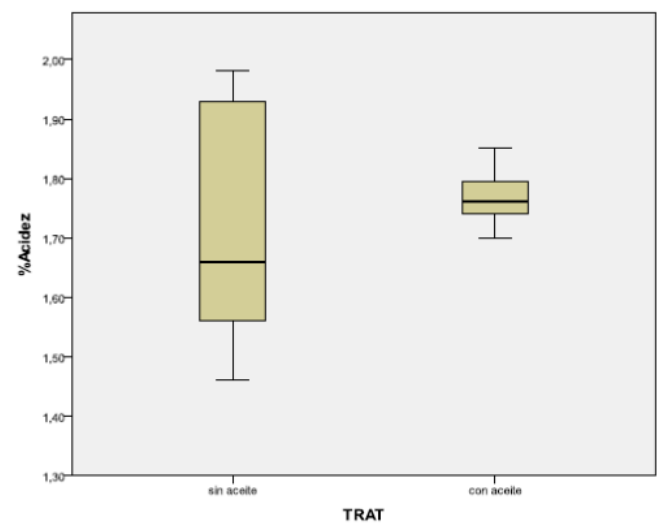

Elaborado por: grupo de investigación 
Los valores inferiores de acidez titulable obtenidos para las muestras control prueban que el proceso de senescencia se está desarrollando. La utilización del aceite esencial de canela como un agente conservante en las uvillas postcosecha permitió una mayor retención de su acidez durante el tiempo de almacenamiento. Este comportamiento probablemente se debe a que el aceite esencial sobre la superficie de la uvilla pudo haber disminuido la velocidad de respiración, con lo que se retardarán las reacciones enzimáticas que provocan la pérdida de la acidez por conversión hacia almidón. El análisis estadístico por el método ADEVA de los resultados obtenidos en los diferentes tratamientos con respecto al parámetro Porcentaje de Acidez Titulable establecen una significancia de 0.040. De acuerdo con los resultados del análisis estadístico (Prueba T) confirma que el tratamiento con aceite esencial de canela aplicado a las muestras de uvilla postcosecha, presenta diferencias significativas con respecto a la fruta sin recubrimiento, ya que el valor de la acidez presenta una disminución menor a la observada en la fruta sin recubrimiento lo que favorece a mantener los atributos de calidad de la Uvilla y por lo tanto incrementa la vida de anaquel de la misma (Tabla5).

Tabla5: Prueba t de muestras independientes para porcentaje de acidez titulable en uvilla

\begin{tabular}{|c|c|c|c|c|c|c|c|c|c|c|}
\hline & & $\begin{array}{l}\text { Prueba de Le } \\
\text { la igualdad d }\end{array}$ & \multirow[t]{3}{*}{ arianzas } & \multirow[b]{3}{*}{$\mathrm{t}$} & \multirow[b]{3}{*}{$\mathrm{gl}$} & \multicolumn{3}{|c|}{ Prueba T para la igualdad de medias } & \multirow{2}{*}{\multicolumn{2}{|c|}{$\begin{array}{c}\text { 95\% intervalo de } \\
\text { confianza para la } \\
\text { diferencia }\end{array}$}} \\
\hline & & \multirow{2}{*}{ C } & & & & & Diferencia de & Error tip. & & \\
\hline & & & & & & (bilateral) & medias & diferencia & Inferior & Superior \\
\hline \multirow[t]{2}{*}{$\mathrm{pH}$} & $\begin{array}{l}\text { Se han asumido } \\
\text { varianzas } \\
\text { iguales }\end{array}$ & 92,228 & 000 & $-2,097$ & 71 & , 040 &,- 05594 & ,02668 &,- 10913 &,- 00275 \\
\hline & $\begin{array}{l}\text { No se han } \\
\text { asumido } \\
\text { varianzas } \\
\text { iguales }\end{array}$ & & & $-1,541$ & 24,904 & 136 & -05594 & ,03630 &,- 13071 &,- 01882 \\
\hline
\end{tabular}

Elaborado por: Grupo de investigación

\section{Recuento de Mohos y Levaduras}

Uno de los factores más determinantes para el mantenimiento de la calidad y la inocuidad de un producto es el factor microbiológico, que se constituye en la respuesta experimental más valiosa en el presente trabajo de investigación. El recuento microbiano de mohos y levaduras se efectuó mediante la técnica de resiembra. Los resultados obtenidos luego de la aplicación del aceite esencial de canela por inmersión a muestras de uvilla presentan un valor inicial de 50UFC/g que disminuye progresivamente hasta alcanzar un valor de 21UFC/g, (Tabla3). Estos resultados se encuentran dentro del límite previsto en el reglamento sanitario de los alimentos que es de 105 
UFC/g (López, 1999) Al comparar los resultados del recuento de mohos y levaduras realizados a la fruta control con los resultados obtenidos al aplicar los tratamientos de aceite esencial de canela como agente conservante permiten establecer que existe un efecto antifúngico del aceite esencial de canela, ya que se puede observar una disminución significativa del número de unidades formadoras de colonias con respecto a las muestras control, por ende se establece también una disminución del riesgo de alteraciones por acción de microorganismos (Gráfico5).

Gráfico5: Mohos y levaduras (ufc/g) en uvillas con y sin recubrimiento de aceite esencial de canela

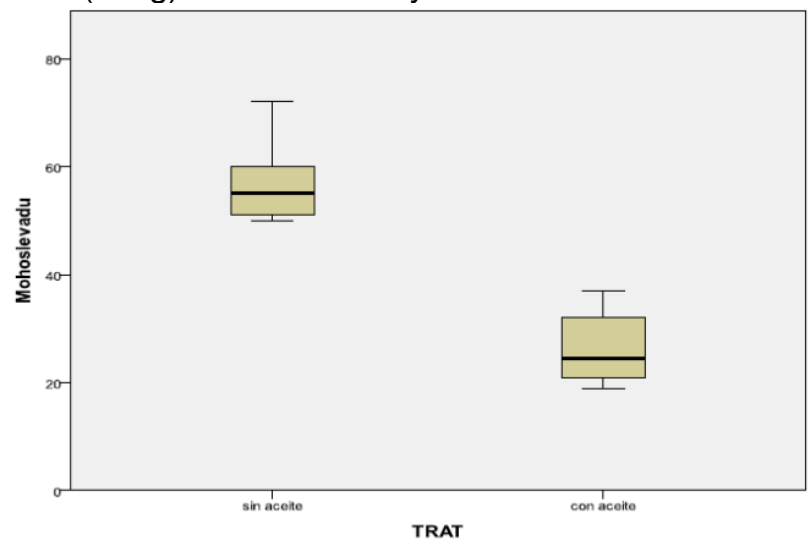

Elaborado por: grupo de investigación

La significancia de estos resultados fue comprobada con la aplicación de un Análisis de Varianza (Tabla 6) en el cual podemos determinar con exactitud que existe una significancia menor a 0.05 entre las frutas control (uvillas sin recubrimiento) y los tratamientos de aceite esencial de canela aplicados a uvillas frescas en concentraciones de 250 y 500 ppm. Estos resultados permiten la aceptar la Hipótesis experimental de que el tratamiento con aceite esencial de canela posee un efecto conservante, ya que con estas condiciones se logra disminuir de una carga microbiana inicial y por ende de las alteraciones del producto por acción de los microorganismos.

Tabla6: Prueba t de muestras independientes para recuento de mohos y levaduras

\begin{tabular}{|c|c|c|c|c|c|c|c|c|c|c|}
\hline \multirow{5}{*}{$\mathrm{pH}$} & & $\begin{array}{l}\text { Prueba de Le } \\
\text { la igualdad d }\end{array}$ & \multirow[t]{3}{*}{ rianzas } & \multirow[b]{3}{*}{$\mathrm{t}$} & \multicolumn{4}{|c|}{ Prueba $T$ para la igualdad de medias } & \multirow{2}{*}{\multicolumn{2}{|c|}{$\begin{array}{l}95 \% \text { intervalo de } \\
\text { confianza para la } \\
\text { diferencia }\end{array}$}} \\
\hline & & \multirow[b]{2}{*}{$\mathrm{F}$} & & & & Sig. & Diferencia de & $\begin{array}{c}\text { Error tip. } \\
\text { de la }\end{array}$ & & \\
\hline & & & & & $\mathrm{gl}$ & (bilateral) & medias & diferencia & Inferior & Superior \\
\hline & $\begin{array}{l}\text { Se han asumido } \\
\text { varianzas } \\
\text { iguales }\end{array}$ & 1,465 & ,230 & 20,074 & 71 & ,000 & 30,278 & 1,508 & 27,270 & 33,285 \\
\hline & $\begin{array}{l}\text { No se han } \\
\text { asumido } \\
\text { varianzas } \\
\text { iguales }\end{array}$ & & & 18,408 & 38,74 & ,000 & 30,278 & 1,645 & 26,950 & 33,605 \\
\hline
\end{tabular}

Elaborado por: Grupo de investigación 


\section{Evaluación Sensorial}

Los resultados de la evaluación sensorial obtenidos de los tratamientos de aceite esencial de canela aplicados a uvillas frescas fueron tomados en periodos de tiempo que van desde 7, 15, 21 y 30 días del almacenamiento en refrigeración $\left(5^{\circ} \mathrm{C}\right)$ y temperatura ambiente $\left(21^{\circ} \mathrm{C}\right)$. Estos resultados se pueden observar en la tabla se encuentran reportados en la Tabla3. Los atributos evaluados fueron color, olor, sabor y textura al tocar. La aplicación de los tratamientos propuestos 250 y 500 ppm de aceite esencial de canela y probados en dos condiciones de temperatura $\left(5^{\circ} \mathrm{C}\right.$ y $\left.21^{\circ} \mathrm{C}\right)$, mantienen las características de color, aroma, textura y sabor en el mismo nivel durante al menos 24 días lo que contrasta con los resultados obtenidos para las uvillas sin recubrimiento que únicamente fueron de 15 días a temperatura ambiente y de 20 días a temperatura de refrigeración.

\section{Conclusiones.}

- El presente estudio permitió comprobar el efecto del aceite esencial de canela sobre uvilla fresca mediante el método de inmersión. La aplicación del aceite esencial de canela conservó las características sensoriales, fisicoquímicas y microbiológicas en la fruta testeada hasta por 30 días, concluyéndose que las concentraciones de 250 y 500 ppm de aceite esencial de canela muestran un efecto conservante sobre uvilla postcosecha.

- Las soluciones de aceite esencial de canela (250 y 500 ppm) utilizadas en el experimento permitieron mantener por más tiempo valores de $\mathrm{pH}$ y acidez similares a la fruta fresca en las condiciones de almacenamiento a temperatura ambiente y refrigeración conservando así la calidad de los productos e incrementando su vida de anaquel.

- El tratamiento con aceite esencial de canela en una concentración de 500 ppm y en temperatura de refrigeración $\left(5^{\circ} \mathrm{C}\right)$ logró incrementar la vida útil de las uvillas frescas hasta por 30 días manteniendo todas las características de la fruta fresca y su inocuidad. Es decir, a mayor concentración de aceite esencial mayor es el tiempo de vida útil

\section{Referencias Bibliográficas.}

Agencia de regulación y control Fito y zoosanitario. (2005). Proyecto BID-FOMIN: Programa para la mitigación de barreras de acceso a mercados bajo ATPDEA. Plagas dudosas de Physalis peruviana en Ecuador. Quito - Ecuador

Agencia de regulación y control Fito y zoosanitario. (2016). Guía de buenas prácticas agrícolas para el cultivo de uvilla. Recuperado de http://www.agrocalidad.gob.ec/wpcontent/uploads/Gui\%CC\%81a-de-BPA-para-Uvilla-1.pdf 
Almanza, P. y Fischer, G. (1993). La uchuva (Physalis peruviana L.) Una alternativa promisoria para las zonas frías de Colombia. Agricultura Tropical. 30 (1):79-87

Álvarez, G., Campoverde, G., \& Espinoza, M. (2012). Manual técnico para el cultivo de uvilla (Physalis peruviana 1) - Universidad Nacional de Loja. Recuperado 4 de febrero de 2019, de https://www.yumpu.com/es/document/read/27634955/folleto-corregidoindduniversidad-nacional-de-loja

Association Of Official Analytical Chemists (A.O.A.C.). Official Methods of Analysis. 16a. Ed. Washington. D.C, A.O.A.C., 2000.

Brito, D. (2002). Agroexportación de productos no tradicionales. Producción de uvilla para exportación. Quito, Ecuador.

Calvo, I. (2009). Cultivo de la uchuva (Physalis peruviana 1)-Mag.go.cr. Recuperado el 17 April 2020, de http://www.mag.go.cr/bibliotecavirtual/AV-0984.pdf.

Corporación Colombia Internacional (CCI). Mora un Cultivo Promisorio. Exótica, mayo 2004, vol. $10, \mathrm{n}^{\circ} 2$, pp. 13-17

Ecuador inicia exportaciones de uvilla hacia Estados Unidos - Ministerio de Producción Comercio Exterior Inversiones y Pesca. (2018). Recuperado de https://www.produccion.gob.ec/ecuador-inicia-exportaciones-de-uvilla-hacia-estadosunidos/

Fao, \& López, A. (2003). Manual Para la Preparación Y Venta de Frutas Y Hortalizas. Roma, Italia: Fao.

Fischer, Gerhard, Almanza-Merchán, Pedro José y Miranda, Diego. (2014) Importancia y cultivo de la uchuva (Physalis peruviana L.). Revista Brasileira de Fruticultura, 36 (1), 01-15. https://doi.org/10.1590/0100-2945-441/13

Gallo, F. Índice de madurez para piña cayena lisa, guanábana, pitaya amarilla y maracuyá. En: Agrodesarrollo. Vol. 4, Nos.1/2 (1993); p. 171-200.

García C., Brito B., Peña. C (2008). Desarrollo tecnológico para el fortalecimiento del manejo postcosecha de la uchuva (Physalis peruviana). Informe final del proyecto Fontagro Ftg. 1403. Corpoica, Iniap, Ciat, Proexant, Cirad. https://repositorio.iniap.gob.ec/bitstream/41000/3275/1/iniapscCD64.pdf

Hernández, L. (2019). Desarrollo de una formulación en polvo a base de Amaranto (Amaranthus cruentus) y Canela (Cinnamomun sp) sabor chocolate (tesis doctoral). Universidad autónoma de Barcelona. 
Martínez, A. (2007). Manual del Cultivo de la Mora de Castilla (Rubus glaucus benth). (1 ed.). Ecuador:

Novoa, R.H., Bojacá, M., Galvis, J.A. y Fishcer, G. (2006). La madurez del fruto y el secado del cáliz influyen en el comportamiento postcosecha de la uchuva, almacenada a $12{ }^{\circ} \mathrm{C}$ (Physalis peruviana L.). Agronomía. Colombia. 24 (1):77 - 86

Nte Inen 1529- 11. 2013. Control microbiológico de los alimentos. Mohos y levaduras viables. Detección. Quito: INEN, 2013.6p.

Nte Inen, 2485.2009. Frutas frescas. Uvilla. Requisitos. Quito: INEN, 2009. 9p.

Prakash, Bhanu \& Singh, Priyanka \& Kedia, Akash \& Dubey, N.K. (2012). Assessment of some essential oils as food preservatives based on antifungal, antiaflatoxin, antioxidant activities and in vivo efficacy in food system. Food Research International. 49. 201-208. 10.1016/j.foodres.2012.08.020.

Rodriguez, M. (2003). Estudio de la conservation de la uchuva (Physalis peruviana L.) utilizando los métodos de atmósfera modificada, refrigeración y encerado. Trabajo de grado. Departamento de Química, Facultad de Ciencias, Universidad Nacional de Colombia, Bogotá.

Shama, G. (2007). Process challenges in applying low doses of ultraviolet light to fresh produce for eliciting beneficial hormetic responses. Postharvest Biology and Technology, 44(1), 1-8. https://doi.org/10.1016/j.postharvbio.2006.11.004.

Stone, H., \& Sidel, J. L. (2004). Introduction to Sensory Evaluation. Sensory Evaluation Practices, 1-19. https://doi.org/10.1016/b978-012672690-9/50005-6

Villada, J. (2010). Conservadores químicos utilizados en la industria alimentaria. Universidad autónoma agraria “Antonio Narro” Monografía Ingeniero en ciencias y tecnología de los alimentos. Recuperado de http://repositorio.uaaan.mx:8080/xmlui/bitstream/handle/123456789/456/61581s.pdf?se quence $=1$

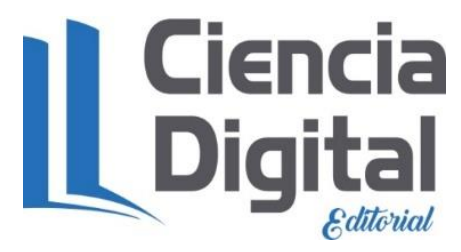


PARA CITAR EL ARTÍCULO INDEXADO.

María Verónica, G. C., Sánchez Herrera, T. E., \& Paredes Peralta, A. V. (2020). Determinación de la capacidad conservante del aceite esencial de canela sobre uvilla (Physalis peruviana) como $\begin{array}{lllll}\text { tratamiento } & \text { postcosecha } & \text { ConcienciaDigital, } & 3(2.1), & \text { 210-230. }\end{array}$ https://doi.org/10.33262/concienciadigital.v3i2.1.1235

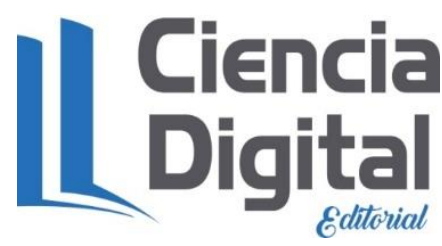

El artículo que se publica es de exclusiva responsabilidad de los autores y no necesariamente reflejan el pensamiento de la Revista Conciencia Digital.

El artículo queda en propiedad de la revista y, por tanto, su publicación parcial y/o total en otro medio tiene que ser autorizado por el director de la Revista Conciencia Digital.
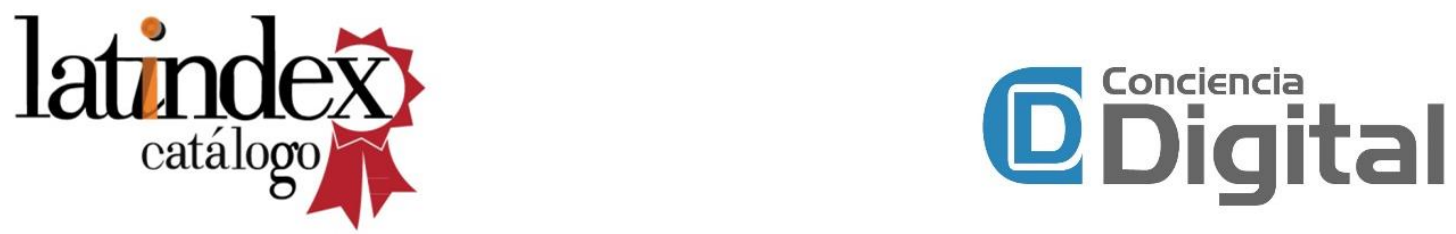